\title{
Brain Tumor Detection by using Fcm Algorithm
}

\author{
S.Kokila, B.Indhu
}

\begin{abstract}
: capable of changing a picture into digital type and it perform operations on image. In image process, input is a picture (may be a video frame or a photograph in any format) and therefore the output is also a picture or the characteristics of the input image. Image process system sometimes considers a picture as a 2 dimensional signal, whereas process. It's one in all the rising technologies, with its branches of application widespread into many domains of business. Image process may be a core analysis in space engineering and it additionally acts as a thrust space in alternative disciplines of applied science. Researchers would like to do perform research in image processing; because it offers real time applications and therefore the results derived from image processing techniques are created. In this paper we have discussed about the greedy snake segmentation, snake contour detection and fcm optimization techniques for segmenting the tumor image, the accuracy level is increased up to $90 \%$ compared with the existing algorithm.
\end{abstract}

Keywords :Image, Signal ,Brain, process, detection, segmentation, fcm, greedy snake..

\section{INTRODUCTION}

There are 2 main ways in image process and that they are:

1. Analog Image process

2. Digital Image process

\subsection{ANALOG IMAGE PROCESS}

Analog image process relates to the modification of a picture through analog or electrical signals. a standard example of this method is that the TV image.

The amplitude levels of the voltage vary let's say the brightness of a picture. Through the appliance of various electrical signals.

The appearance of the displayed image is changed (or) altered. The brightness and distinction levels within the image are altered with the help of each the controls obtainable on amplitude and therefore the reference of the video signals in an exceedingly idiot box.

\subsection{DIGITAL IMAGE PROCESS}

Digital image process usually denotes the procedure of process a two- dimensional image by a electronic computer. A digital image is comprised of an array of real variety symbolized by a finite number of bits.

The image would be regenerate to a digital type mistreatment a device, say scanner, and then the any process upon the image is completed. It also can be mentioned because the numerical representations of the pictures to change a sequence of operations to derive a desired result.

Revised Manuscript Received on April, 042020.

* Correspondence Author

Mrs. S.Kokila*, Assistant Professor, Department of Electronics and Communication Engineering, Vivekanandha College of Engineering for Women

Ms. B.Indhu, PG Scholar, Department of Electronics and Communication Engineering, Vivekanandha College of Engineering for Women

(c) The Authors. Published by Blue Eyes Intelligence Engineering and Sciences Publication (BEIESP). This is an open access article under the CC BY-NC-ND license (http://creativecommons.org/licenses/by-nc-nd/4.0/)
Digital image process commences the process with one image and at last, a reformed version of an equivalent image is obtained.

\subsection{APPLICATIONS OF THE IMAGE PROCESS}

- Visualization - to look at the objects that don't seem to be visible to the human eye.

- Image sharpening and restoration - to come up with a higher image.

- Image retrieval - to spot and retrieve sure valuable data for the interested image.

- Measurement of pattern - Measures varied objects of various geometric shapes in a picture.

\subsection{DIAGNOSIS OF A GROWTH}

Various test is done for the diagnosis of the tumor, doctor examine the mental stability, muscle strength and nerve operate by the physical examination, various other test can also be done

- Audiometric: diagnose the hearing ability of the person and it is done by audiologist, detects, tumor from inner ear to the brain (e.g., acoustic neuroma), which is non cancerous.

- Endocrine analysis: detects the abnormal level of blood pressure in pituitary. Which reregulate the blood flow in the body (e.g., Cushing's disease).

- Neuro-opthalmologist: finds out the visual imbalance in the body, neuro-opthalmologist integrate both nerves and visual.

- Spinal tap is performed to look atliquid body substance for growth cells, proteins, infection, and blood.

\subsection{IMAGING TESTS}

Tumor can be detected by using CT and MRI. The three dimensional image of the patients can be obtained by both CT and MRI. Mostly in hospitals MRI three dimensional images have been used because of the easy identification of the tumor. The image is segmented layer by layer. The brain inside the rigid skull is clearly viewed in CT when compared to MRI. The MRI uses radiofrequency and force field to get the clear image. The dye is inserted into the blood before testing the patients. Cross sectional images are obtained by the CT scan. Intravenous contrast agents are used for the diagnosis of lesions in the bone, iodine is mostly used as the contrast agent. The below diagram shows the image of benign and malignant which is obtained by the MRI and shown in fig (1.1) 


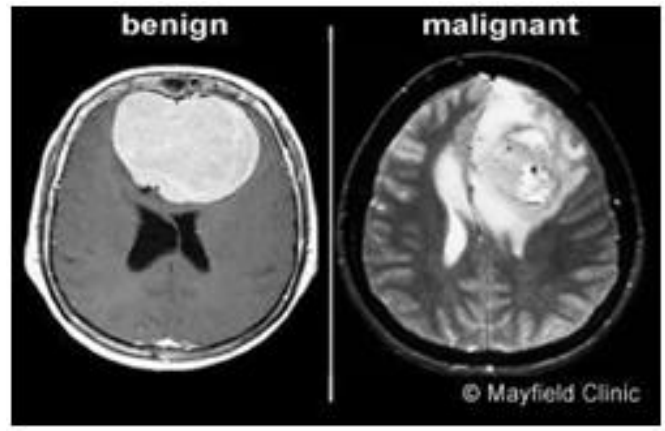

Fig 1.1 imaging scans of a benign and malignant tumor

\subsection{ORGANIZATION OF REPORT}

The setup of this report is as follows: chapter 3 reviews the prevailing ways. Computer code specification is roofed in chapter4. The implementations and a discussion of the results of this project ar bestowed in chapters5. Finally, the expected conclusion of the planned module is provided in chapter6.

\section{LITERATURE SURVEY}

\subsection{SURVEY OF PAPERS}

\section{EDGE DETECTION TECHNIQUE}

Edge detection has its own name within the domain of image process. The sides recognized by edge detection techniques area unit typically non - continuous. To phase an object or a part of need from a picture, one needs closed region boundaries. The expected edge area has the unit boundaries that lie between such objects. So, edge is primarily based on detection which supports image segmentation. One such edge detection technique was projected by cagy [3], which utilizes optimum smoothing filter to preserve the sides

\section{THRESHOLDING TECHNIQUE}

The simplest technique of image segmentation referred to as the thresholding technique was introduced by Shapiro et $\mathrm{al}[10]$. The operation of the tactic is predicted, that supports the conversion of gray-scale image to binary image. The most objective of this technique is to settle on the edge price (or values once multiple-levels area unit selected), most entropy technique, otsu's method(maximum variance) and k-means agglomeration area unit the foremost frequent thresholding techniques employed in industries. Threshold price act as mid-value, and input prices higher than or below the nominative threshold value area unit finally displayed.

\section{COMPRESSION BASED TECHNIQUE}

Compression primarily based ways explicate that the optimum segmentation helps to reduce the general attainable segmentations, that more favor the reduction of code length of the info [2, 6, 14]. The connection between these 2 ideologies is that the segmentation tries to spot patterns gift in an input image and it will closely monitor the regularity of the gift within the image, which might be employed in compression.

\section{HISTOGRAM-BASED TECHNIQUE}

Histogram primarily based technique manufacture economical segmentation ends up in comparison with different segmentation ways as a result of they need just one entry through the pixels $[8,12]$. By this method, a bar graph is calculated from the entire pixels gift within the image.

The peaks and valleys gift within the bar graph area unit won't assign the clusters within the image. Color or intensity price are often wont to discover the peaks and valleys. On reiterative application of bar graph primarily based segmentation technique, the input image is partitioned off into many smaller clusters. This procedure is perennial with the formation of smaller and smaller clusters till all the clusters area is unit shaped. Bar graph procedures are often wiped out multiple sides once multiple frames area unit is being processed. Constant procedure adopted in process one frame may also be applied to method multiple frames. This method is followed because the final results area unit is united, in order that the identification of peaks and valleys is created simple and located to be clearly distinguishable. The bar graph methodology may also be applied on one component.

\section{REGION-GROWING TECHNIQUE}

Region Merging technique [9] is an iterative process. Also, this technique utilizes a collection of seeds as input. The seed in every object is segmented. The region in area unit adult iterate by the scrutiny of all unallocated or nongrouped neighbor pixels to the regions. The component with the littlest differential price measured during this procedure is allotted to several regions. This method take place till all pixels area unit is allotted. The extra input is denoted as seed. In segmented output the area unit is entirely passionate about the selection of seeds. It is totally different from seeded region growing, because it uses marginal differential price that is smaller amount than a predefined threshold, supported that the component is else to the actual region. If this criterion isn't met, then the component is taken into account to differ from all the present regions and a replacement region is formed out with this component.

\section{SPLIT-AND-MERGE TECHNIQUE}

Split and merge segmentation depends on quad V' tree partition. It is additionally referred to as the Quadra segmentation. This technique begins with foundation of tree that represents the entire input image. If non uniformity is discovered, then the tree structure more splits into four sections so on. On the opposite hand, if four non-squares area unit homogenous, they'll be classified as one connected part. The node gift within the tree structure could be a segmental node. This method continues repeatedly till no more splits or merges may be created. Once the spilt-and-merge methodology is influenced by a special organization sequence, then the implementation of this method is simple and therefore the methodology needs lesser machine time for execution.

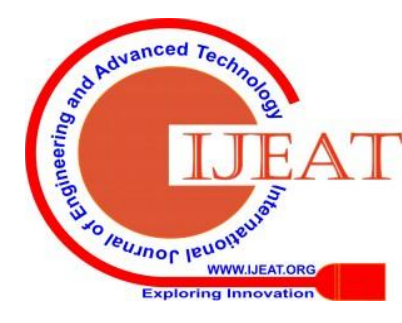




\section{PARTIAL EQUATION BASED TECHNIQUE}

An image is metameric by numerically finding a partial equation (PDE) - primarily based method [4]. Curve propagation could be a common in PDE primarily based methodology , by that varied applications associated with object extraction, object following, stereo reconstruction, etc square measure performed.

The prime plan of victimization this system is to seek out associate degree initial curve towards rock bottom potential of a value operate, wherever its description relates the task to be processed. For many of the converse issues, diminution of the price operate is of a lot of importance and it proposes sure smoothness constraints on the answer,

\section{PARAMETRIC METHODOLOGY}

Parametric methodology depends upon parameterization of the contour in keeping with pictures and internal terms [7]. Such techniques square measure quick enough and economical in manufacturing segmentation results; but the initial "purely parametric" formulation is mostly critiqued for its limitation in selecting the sampling strategy, topology changes, addressing downside in higher dimensions, the inner geometric properties of the curve, etc. recently "discredited" formulations are evolved to deal with the constraints incurred throughout high potency maintenance. Within the on top of aforesaid cases, energy diminution is mostly organized

\section{LEVEL SET METHODOLOGY}

Osher and Sethian [11] planned tier set methodology to trace moving borders or interfaces unfold across numerous imaging domains. It is accustomed effectively handle the matter of curve or surface propagation in a picture. The prime plan is the representation of the developed contour by a signed operates, the zero level relates the particular contour.

Victimization depends upon the motion equation of the contour, an analogue flow can be derived easily for the unclear surface, and the consecutive is applied to the zerolevel

which illustrate the propagation of the contour fast walk methodology. Quick walk methodology has been employed in image segmentation by Forcade et al,[5] because the model utilizes each positive and negative propagation speed values for the segmentation of a picture. This approach may also be known as the generalized quick walk methodology.

\section{GRAPH PARTITIONING METHODOLOGY}

Graph partitioning is based on the economical image segmentation [15]. The input image is described as associate degree rudderless weight primarily based graph. The difference between the neighborhood picture elements is sometimes assessed with the assistance.

The image described graph structure is later partitioned off because the same procedure followed to the model clusters. Partition of the pixels represent the outputs derived from the rule and is claimed to be a metameric object of the image. a number of the often used algorithms that lie beneath this

- normalized cuts,

- random walker,

- minimum cut,

- iso-constant quantity partitioning and minimum spanning tree-based segmentation.

\section{WATERSHED TRANSFORMATION}

Watershed segmentation is an region based method and an mathematical morphological. Watershed transformation depends on the gradient magnitude of a picture that acts as a topographical surface [13].Formation of watershed line is done by the highest gradient magnitude, and that they denote the region boundaries. Water positioned on any picture element that is encircled by a typical watershed line. Watershed transformation technique is more faster and accurate. An image is segmented based on the rigid and valleys. Catchment basin is an decomposition of image for the $3 \mathrm{D}$ representation of an image. The basins are separated by the watersheds. The disadvantage in watershed segmentation is an noisy image which occur due to the decomposition of the image. The overlapping and noise of the image can be minimized by using the merging basins technique.

\section{SEMI-AUTOMATIC SEGMENTATION}

Semi automatic segmentation is useful for segmenting the large database, this is done after the manual diagnosis, work and it's known as the seed primarily based region growing rule. Initial level ROI is chosen employing a click which region or voxel act because the seed purpose supported that the segmentation is performed. TumorCut: segmentation of brain tumors on distinction. An enhanced MRI picture for radio surgery applications [1] is capable of segmenting solely distinction increased $\mathrm{T} 1$ weighted pictures. The usage of the growth cut rule is restricted towards $\mathrm{T} 1$ distinction increased pictures and not upon alternative modalities of brain pictures. Growth detection is completed victimization the essential principle of Region of Interest (ROI) and needs manual help for segmentation procedures.

\section{TRAINABLE SEGMENTATION}

Most segmentation methods depend on the shade facts of pixels present in a photograph. While performing image segmentation, humans use a much wider records than coloration records. But, enforcing this factsthis recordsrecords could need ample computation time and massive knowledge primarily based data, that square measure currently now not handy. There square measure trainable segmentation approaches, which may model some of this datafactsstatistics and can manufacture higher results compared to standard knowledge based ways. Neural Network segmentation relies upon on procedure little regions of a photo victimization a artificial neural community or a set of neural networks. As soon as such initial level manner, the choice-making mechanism mark the areas of a image consequently to the popularity offered by using the neural network.

\section{PROPOSED WORK}

\subsection{INTRODUCTION}

Cancer in the brain is a deviation of normal tissue. The most common and primary brain tumors are benign and malignant.30 \% of the brain tumor is occurred by meningioma which occurs in any tissue in the brain, the skull is enclosed by the brain is very hard, the detection of tumor inside the skull is very difficult

Published By:

Blue Eyes Intelligence Engineering DOI: 10.35940/ijeat.C6561.049420

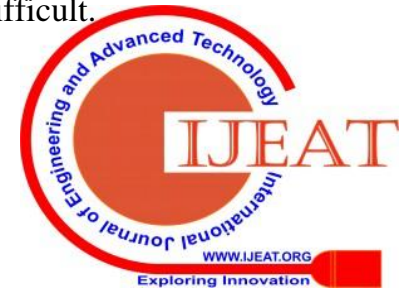


The meningioma occur in the Parasagittal, Olfactory groove, Clivus, Suprasellar, Foramen magnum and Cerebellar, in the brain, the meningioma occurs in different size, shapes and intensities. Glioma is the cancerous growth which occurs in the spinal cord and cerebral part. Early detection helps to find the type of treatment and accurate treatement can be done so that death rate can be reduced. The background minization eliminates the nontumor region, here segmentation is done by using greedy snake and fcm and finally tumor is detected.

\subsection{BLOCK DIAGRAM}

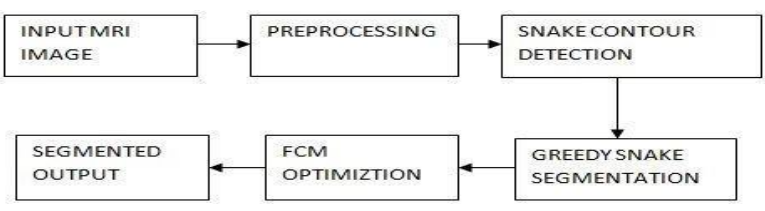

Fig 3.1 block diagram

\subsection{PREPROCESSING}

The main goal of preprocessing is to counterpoint the visual look of the pictures. Preprocessing chiefly aims to get rid of the clamor, stabilising the intensity of the pictures and clear the artifacts. Image preprocessing is that the technique of enhancing the image knowledge before pictures sometimes comprises blurring and noises. The filter is mostly used for removing the noises in which it does not affect the important properties of an image. It is

$$
\mathrm{I} 2(\mathrm{x}, \mathrm{y})=\operatorname{median}\{\mathrm{I} 1(\mathrm{x}, \mathrm{y}),(\mathrm{x}, \mathrm{y}) € \mathrm{~W}\}
$$

where $\mathrm{x}$ represents a three $*$ three neighborhood focused round the location $(\mathrm{x} ; \mathrm{y})$ within the image

In general, image preprocessing is administrated in anyone of the subsequent forms. The disadvantage of preprocessing is which does not reduce noise and artifacts in the CAD system

Generally preprocessing is done by

- Image resampling

- Contrast improvement

- Noise removal

\subsubsection{IMAGE RESAMPLING}

Altering the picture element dimensions of the image is termed resampling. Image resampling could be a procedure to rework a sampled image from one coordinate to a different. Victimization the mapping operate of the dimensional transformation, the 2 coordinate systems are correlative to every different. The reverse mapping operate is applied to the output picture element, in order that the obtained 'resampling picture element' is reversed to get the initial input pixel. In some cases, the resampling picture element doesn't match with the input picture element. so as to beat this, an identical domain should be created for the input picture element and therefore the vary of the mapping

\subsubsection{CONTRAST improvement}

To make the image additional appropriate for definite procedure process. The magnetic resonance imaging noises and rarity are removed by using median filter. Median otherwise known as low level abstraction techniques

applications, distinction improvement should be used. It improves the visibility and therefore the transparency of the image and therefore the original image is additional acceptable to method the pc.As the image values of the low distinction pictures ar extreme, the distinction improvement stretches the intensity of the picture element. Commonly, the image will have poor applied math vary or untruth of pixels either because of quality of the imaging devices or the intense external conditions throughout acquisition method.Among the assorted distinction improvement techniques, bar chart modification procedure is extensively used due to its easiness and effectiveness. the method of bar chart leveling is to stretch the intensity of the input image to make a regular distribution in order that the active vary of the image is totally disheartened.Local difference Stretching (LDS) could be a boosting methodology to regionally modify the intensity price of a picture each in darkest and brightest a part of the image at the same time.

The LDS is enforced victimisation window construct and therefore the center is set from the subsequent expression:

$$
\operatorname{MPx}(\mathrm{i}, \mathrm{j})=255 * \frac{\left[\mathrm{MP0}\left(\mathrm{i}_{i} \mathrm{j}\right)-\mathrm{MPmin}\right]}{\left[M_{\max }-M_{\min }\right]}
$$

where

$\operatorname{MPx}(i, j)$ represents center price of picture element

$\mathrm{x} \operatorname{MPo}(\mathrm{i}, \mathrm{j})$ represents center price of origin

MPmin represents minimum price of all pixels

MPmax represents most price of all pixels

\subsection{MEDIAN FILTER}

Median filter is a noise removing technique or non linear digital filtering technique which is used for removing noise from an image. Median filter is used in preprocessing step to increase the quality, intensity and removing noise without affecting the edge properties of an image. The operation of median is done by replacing the neighbors window by the median and is called the window. The disadvantage in median filter is the boundary issues, missing window of an image and finding the median value for each window, the critical factor is very low. The histogram medians are used when the whole number in the signal is used. Median filter is also used in smooth patches for the reduction of noise and plays an major role so it is called as linear Gaussian filtering. It redue the noise of speckle and salt and pepper noise.

\subsubsection{BOUNDARY PROBLEMS}

Boundary detection techniques are

- $\quad$ Pixel based technique

- $\quad$ Statistic based technique

- Transform based technique

- Feature based technique

- Histogram based technique

The image is different from one to another based on the that the technique is applied

Edge detection is also known as boundary detection and used in the application of image processing

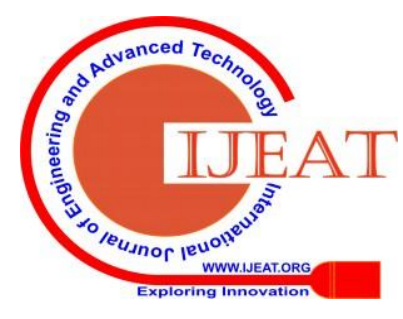




\subsubsection{EDGE PRESERVATION PROPERTIES}

Median filtering is also known as smoothing technique, edges in an image is very important and plays a vital role because it contain the important information of an image, the edges gives the shape of an image.

It is mainly used in data extraction and image segmentation which helps to reduce the noise and blurriness of an image. The value of the neighborhood pixel can be changed by the median filter, so that it is used in the patch smoothening technique which reduces noise Due to this, median filter is widely used in digital image processing.

\subsection{SNAKE OR ACTIVE CONTOUR DETECTION}

The active contour have the following two steps,

(a) Level I morphological reconstruction

(b) Level II morphological reconstruction

\subsubsection{LEVEL I \& II MORPHOLOGICAL RECONSTRUCTION}

Level I morphological reconstruction consists of background minization, the first step in morphological reconstruction is done by erosion and second stage is done by dialation.

The erosion eliminate the non tumor region and maintains the edge properties.

The dilation enlarge the eroded image. The mask is formed by the erosion in which the original size is reduced for example the image has an size of $256 * 256$ and reduce the size of $3 * 3$. The erosion will remove the pixel in an image. The dilation will add the pixel in an image.

The properties in erosion are

- Translation invariant

- Anti extensive

- Distributive

- Commutative

- Set intersection

Dilation and erosion operation depends on the neighborhood pixel. Erosion and dilation are used for smoothing the edges of an image, noise and small object is also removed Erosion is the first step to occur and it is followed by dilation. The erosion operation occurs if the foreground value is maximum than the background value and dilation operation is occurred if the foreground pixel value is minimum from the background pixel value, various function used for the dilation operation to perform are

- Imopen is the function used for the beginning the operation of erosion and dilation

- Imclose is the function used the ending the erosion and dilation operation

- Bwhitmiss is the function used for preserving the pixel in the digital image

- Imtophat is the function used for the conversion of binary image into contrast image and man

Dilation expands associate object to the nearest pixels of the neighborhood. Normally, dilation is employed to fill little holes and slender gulfs in objects. If the initial size has to be preserved, then dilation is combined with erosion and is explained in next segment.
Erosion shrinks the item. Erosion of a picture is that the operation of assignment to every pixel the minimum price found over a locality of the corresponding pixel whereas dilation is that the operation of assignment to every pixel the most price of the neighborhood.

The structuring component may perform 2 variables that specify the required native grey level property. The worth of the structuring component is additional or ablated for calculative most or minimum levels within the neighborhood.

3.6 APPLICATION OF MORPHOLOGICAL IMAGE

FILTERING

- Boundary extraction

- Convex hull

- Thinning

- Morphological filtering

- Pruning

- $\quad$ skeletons

\subsection{HITS OR MISS TRANSFORMATION}

Hits or Miss transformation is an morphological tool for shape detection. The origin is located at the center of an image. It is an mathematical morphology. The output of hits or miss transformation consists of first structuring element and second structuring element, the input image consists of foreground and background in which first structuring element is fitted to the foreground, second structuring is lost.
3.8 APPLICATIONS
$\mathrm{OF}$ MORPHOLOGY
- Morphological smoothing
- Morphological gradient
- Top-hat\& bottom-hat segmentaion
- Textural segmentation
- Granulometry

GRAY

LEVEY

Morphological smoothing performs opening and closing operation

Morphological gradient performs erosion and dilation operation

Top \& bottom hat segmentation have dark and light objects

Dark and bright background contains the dark and bright background respectively.

Textural segmentation detects the boundary of an image Granulometry performs the size distribution properties

3.9 GREEDY SNAKE SEGMENTATION

A greedy algorithmic program is any algorithmic program that follows the problem-solving heuristic of constructing the regionally best alternative at every stage with the intent of finding a world optimum. In several issues, a greedy strategy doesn't typically turn out associate best answer; however a greedy heuristic might yield regionally best answers that approximate a globally best solution in an exceedingly cheap quantity of your time.

For example, a greedy strategy for the roadman drawback (which is of a high machine complexity) is that the following heuristic: "At every step of the journey, visit the closest unvisited town."

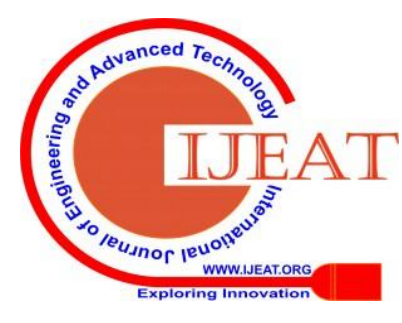


This heuristic doesn't will realize a best answer, however it terminates in a or during a or in associate exceedingly in a very $\}$ cheap range of steps; finding an best answer to such a fancy drawback generally needs immoderately several steps. In mathematical improvement, a greedy algorithm optimally solves combinatorial issues having the properties of matroids, and provides constant-factor approximations to improvement issues with sub modular structure. Snakes or active contour may be a curve that moves at intervals a image, wherever there's minimum energy. The snake moves and tries to seek out the boundaries of a part of interest beneath the influence of external and internal forces. The initial contour of the snake will be calculable from the boundary of expanded image. The image is metameric victimization the initial contour calculable from the boundary of the region in expanded image to get the greedy snake metameric image.

\subsection{FUZZY -C-MEANS CLUMP IMPROVEMENT}

The FCM clump works by assignment membership to every

background), the quantity of clusters cis set to a pair of. The FCM algorithmic program uses the pixels that are boxed within the region metameric by Greedy snake segmentation algorithmic program.

The sides of the Greedy snake segmentation algorithmic program are correct since the sting pixels might or might not belong to the neoplasm port. The FCM algorithmic program classifies the whole pixels boxed within the region metameric by Greedy snake algorithmic program into 2 clusters, wherever one cluster contains the pixels of the neoplasm tissues whereas the opposite cluster contains the pixels of the non neoplasm region.

\section{RESULT AND DISCUSSION}

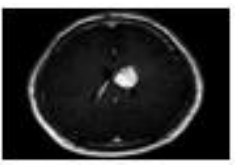

Fig 4.1 Input image

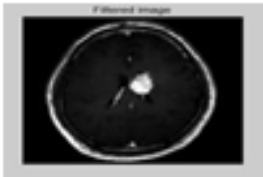

Fig 4.2 filtered image

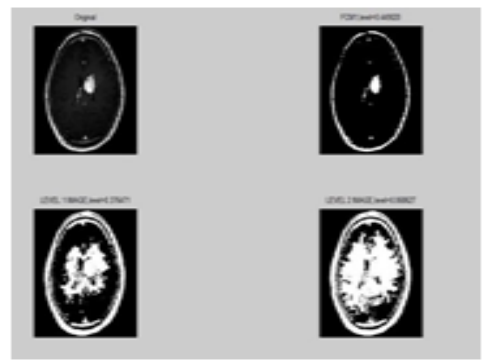

Fig 4.3 output of level of morphological reconstruction

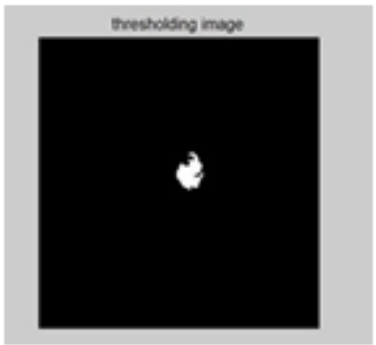

FIG 4.4 OUTPUT OF THRESHOLDING

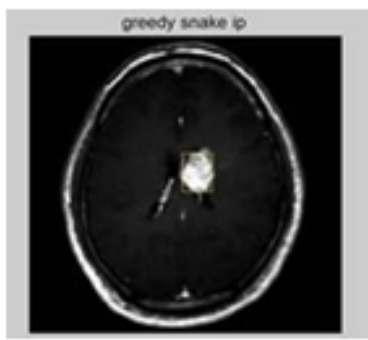

FIG 4.5 INPUT OF GREEDY SNAKE

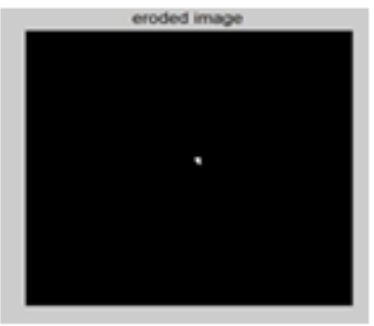

FIG 4.6 OUTPUT OF EROSION

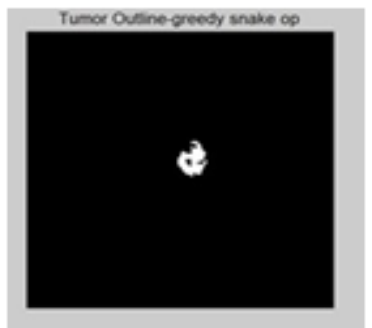

FIG 4.7 OUTPUT OF EROSION

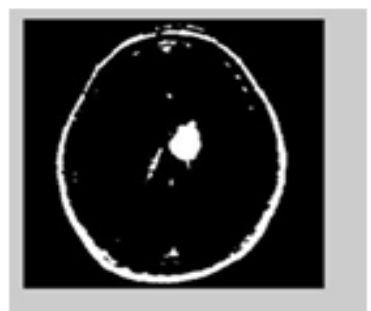

FIG4.7 OUTPUT OF FCM

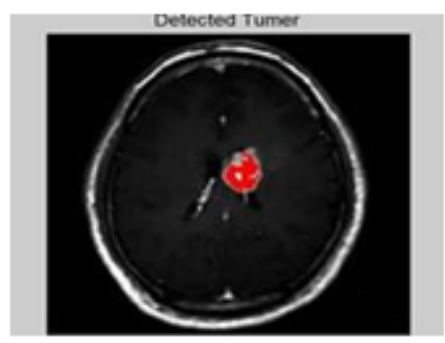

FIG 4.8 TUMOR REGION IS DETECTED

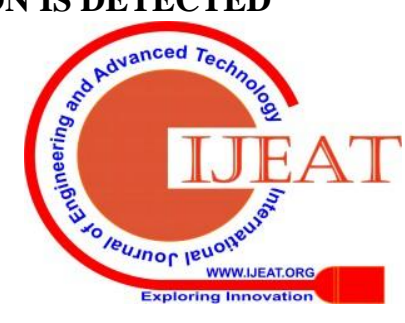




\section{CONCLUSION AND FUTURE WORK}

\subsection{CONCLUSION}

This paper detects the tumor of the MRI image by using greedy snake and fcm optimization technique. The thresholding provides the mask to the input image and the size is reduced. Preprocessing is used for eliminating the noise and blurriness of the image after preprocessing two levels of morphological reconstruction is used, the tumor detected area is indicated in red color.

\subsection{FUTURE WORK}

Volumes of tumor and volumes of traditional tissue regions are often assessed, wherever bottom exploration has been

The accuracy of the tumor are often redoubled by exploitation Mathematical morphological technique rather than greedy snake algorithmic rule

\section{REFERENCES}

1. Andac Hamamci, Nadir Kucuk, Kutlay Karaman, Kayihan Engin and Gozde Unal, Tumor-Cut: Segmentation of Brain Tumors on Contrast Enhanced MR Images for Radiosurgery Applications, IEEE transactions on medical imaging, vol. 31 (2011), 790 - 804.

2. Burt. P and Adelson, E, The Laplacian Pyramid as a Compact Image Code, IEEE Transactions on Communications, 31 (4) (1983), 532-5

3. Canny. J, A computational approach to edge detection, IEEE Trans. Pattern Analysis and Machine Intelligence, Vol 8 (1986), 679-714.

4. Caselles. V, Kimmel. R and Sapiro. G, Geodesic active contours,International Journal of Computer Vision, 22(1) (1997), 61-79.

5. Forcade Nicolas, Le Guyader, Carole Gout, Christian (July), Generalized fast marching method: applications to image segmentation, Numerical Algorithms, 48 (1-3) (2008), 189-211, $<$ doi:10.1007/s11075-008-9183-x>.

6. Hossein Mobahi, Shankar Rao, Allen Yang, Shankar Sastry and Yi Ma, Segmentation of Natural Images by Texture and Boundary Compression, International Journal of Computer Vision (IJCV), 95 (1) (2011), 86-98.

7. Kass. M, Witkin. A and Terzopoulos. D, Snakes: Active contour models, International journal of computer vision, 1 (4) (1988), 321331.

8. Ningning Zhou, Tingting Yang, and Shaobai Zhang, An Improved FCM Medical Image Segmentation Algorithm Based on MMTD, Computational and Mathematical Methods in Medicine, Volume 2014, Article ID 690349, http://dx.doi.org/10.1155/2014/690349.

9. Nock. R and Nielsen. F, Statistical Region Merging, IEEE Transactions on Pattern Analysis and Machine Intelligence, Vol 26, No 11 (2004), 1452-1458.

10. Shapiro, Linda G. \& Stockman, George C, Computer Vision, Prentice Hall, ISBN 0-13-030796-3.

11. Osher. S and Sethian.J.A, Journal of Computational Physics, 79 (1988), 12-49.

12. Pearson. K, Contributions to the Mathematical Theory of Evolution. II. Skew Variation in Homogeneous Material, Philosophica Transactions of the Royal Society

13. A: Mathematical, Morphology in Image Processing (Ed. E. R. Dougherty), (1993), 433-481.

14. Shankar Rao., Hossein Mobahi., Allen Yang., Shankar Sastry and Yi Ma., Natural Image

15. Segmentation with Adaptive Texture and Boundary Encoding, Proceedings of the Asian Conference on Computer Vision (ACCV) (2009), H. Zha, R.-i. Taniguchi, and S. Maybank (Eds.), Part I, LNCS 5994 (2009), 135-146.

16. Zahn. C.T, Graph-theoretical methods for detecting and describing gestalt clusters, IEEE Transactions on computers, Vol 20, No. 1(1971), 68-86 